\title{
Comparación de distintas estrategias para la modificación de la actitud hacia el consumo de drogas en escolares
}

\author{
José Pedro Espada Sánchez ${ }^{1}$, Olga Hernández Serrano ${ }^{2}$, \\ Mireia Orgilés Amorós ${ }^{3}$, Xavier Méndez Carrillo ${ }^{4}$ \\ ${ }^{123}$ Universidad Miguel Hernández \\ ${ }^{4}$ Universidad de Murcia
}

\section{España}

Correspondencia: José Pedro Espada Sánchez. Departamento de Psicología de la Salud. Universidad Miguel Hernández. Campus de Elche. Avda. de la Universidad, s/n. C.P.-03202. Elche, Alicante (España). E-mail: jpespada@umh.es

(C) Education \& Psychology I+D+i and Editorial EOS (Spain) 


\section{Resumen}

Introducción. La evaluación de programas escolares de prevención de drogodependencias indica distintos grados de eficacia según la estrategia utilizada. El objetivo de este trabajo es analizar distintos tipos de intervención preventiva para modificar la actitud hacia las drogas en adolescentes.

Método. Se utilizó un diseño cuasi-experimental con cinco condiciones: dos programas de prevención específica (Programa Saluda y Programa Life Skills Training), un programa de Educación para la salud, un grupo de Atención Placebo y un grupo de control sin intervención.

Resultados. El Programa Saluda y el programa Life Skills Training mejoraron los resultados frente al grupo de Atención Placebo y el grupo sin intervención sobre la intención de consumo de alcohol. El análisis de los tamaños del efecto en la actitud ante las drogas indica que el programa Saluda produce mayores cambios actitudinales en comparación con los demás grupos.

Discusión y Conclusiones. Se discuten los hallazgos alcanzados y las implicaciones en futuras investigaciones sobre el efecto de los programas en la actitud hacia las drogas y su consumo.

Palabras Clave: actitudes, adolescencia, drogodependencias, evaluación de programas, prevención escolar.

Recibido: 11/05/10 Aceptación Inicial: 12/05/10 Aceptación Definitiva: 09/09/10 


\title{
A comparison of different strategies for modifying attitudes towards drug use in schoolchildren
}

\begin{abstract}
Introduction. The evaluation of school-based programs to prevent substance abuse point out varying degrees of effectiveness depending on the strategy used. The aim of this paper is to analyze different types of preventive intervention to change the attitude towards drugs in adolescents
\end{abstract}

Method. A quasi-experimental design was conducted with five conditions: two specific prevention programs (Program Saluda and Life Skills Training Program), a Health Education program, an Attention Placebo group and a control group without intervention.

Results. Program Saluda and Life Skills Training program reached higher changes in attitudes and intention of drinking compared to the Placebo Condition and the group without intervention. The analysis of effect sizes in the attitude to drugs indicates that program Saluda produces greater attitudinal changes compared with the other groups.

Discussion and Conclusions. The implications for future research on the impact of programs in the attitude towards drug use are discussed.

Keywords: Attitudes, adolescence, substance abuse, program evaluation, school-based prevention.

Received: 05/11/10 Initial Acceptance: 05/12/10 Definitive Acceptance: 09/09/10 


\section{Introducción}

El consumo de drogas se encuentra muy extendido entre los adolescentes españoles, que se inician en el consumo a edades cada vez más tempranas (Plan Nacional sobre Drogas, 2008). El abuso de sustancias conlleva numerosas consecuencias negativas en diferentes áreas de la vida del adolescente, como pueden ser problemas de rendimiento académico, embarazos no deseados, enfermedades de transmisión sexual, problemas de aprendizaje y memoria, accidentes de tráfico, problemas legales y afectivos, muertes por suicidio y problemas familiares y económicos (De Bellis et al., 2000; Espada, Méndez e Hidalgo, 2000; Masten y Faden, 2008; Observatorio Español sobre Drogas, 2008).

En España, según los datos de la Encuesta Estatal sobre Uso de Drogas en Enseñanza Secundaria (Observatorio Español sobre Drogas, 2008), el alcohol y el tabaco son las sustancias más consumidas con tasas del $58 \%$ y del $32.4 \%$ respectivamente. La tendencia respecto a años anteriores indica que el consumo de alcohol se mantiene estable, aunque aumenta la frecuencia de los episodios de embriaguez. En el caso del tabaco el consumo experimental ha aumentado y se mantiene el consumo diario. A estas dos sustancias le sigue en frecuencia el consumo de cannabis con una tasa del $20.1 \%$, seguido por otras drogas ilegales de menor prevalencia en este intervalo de edad.

Las evidencias sobre la eficacia de los programas de prevención escolar de consumo de sustancias muestran resultados diversos. Según datos de EDDRA (European Monitoring Centre for Drugs and Drug Addiction, 2006), de un total de 52 programas preventivos realizados en España, la mayoría no incluyen una evaluación de resultados, y entre los programas evaluados la mayoría presentan problemas metodológicos. Salvador y Martínez (1997) analizaron 503 programas de prevención del consumo de drogas, hallando que aproximadamente el 60\% de los programas incluía algún tipo de evaluación, en la mayoría de los casos, evaluaciones con importantes limitaciones.

Según las revisiones cuantitativas, el impacto de los programas preventivos españoles en general es consistente pero limitado, siendo el tamaño del efecto pequeño, presentando una baja eficacia y diversas deficiencias metodológicas (Espada et al., 2002). Los resultados alcanzados en España en este ámbito son similares al de otros países, lo que incita a continuar progresando en los estudios sobre este campo de trabajo. 
Entre los modelos teóricos en los que se basan los programas de prevención escolar, la Teoría de la Acción Razonada (Fishbein y Ajzen, 1975) destaca el papel de la intención conductual sobre el consumo, que a su vez está influida por las actitudes hacia el consumo y la norma subjetiva sobre el mismo. Las actitudes se encuentran determinadas por las consecuencias esperadas del consumo respecto a beneficios y costes. Teniendo en cuenta este modelo, distintos estudios resaltan el papel de la actitud hacia las drogas como un factor que influye en el consumo (Colder, Campbell, Ruel, Richardson y Flay, 2002; Golberg, Halpern-Felsher y Millstein, 2002; Grube y Agostinelli, 1999; Turrisi, Wiersma y Hughes, 2000). Una actitud positiva hacia el uso de sustancias es un importante predictor del inicio del consumo de alcohol y otras drogas, y por tanto es una variable a considerar en la evaluación de los efectos de los programas preventivos (Espada, Méndez, Griffin y Botvin, 2003). Asimismo, el bajo consumo se ha asociado con unas expectativas negativas por parte de los individuos hacia el consumo (Grube y Agostinelli, 1999). Por su parte, Hawkins, Catalano y Miller (1992) indican que las actitudes de rechazo a las normas de la sociedad y las actitudes favorables hacia el consumo de sustancias suponen un factor de riesgo frente al consumo entre los adolescentes. Existen estudios que han relacionado las actitudes favorables al consumo junto con una percepción del mismo vinculado a una cierta transgresión de las normas sociales, como predictoras de conductas delictivas o predelictivas durante la adolescencia (Cava, Musitu, y Murgui, 2006).

El objetivo principal de este trabajo es analizar en qué medida las intervenciones preventivas logran modificar la actitud hacia las drogas en una muestra de adolescentes. Se pretende también comparar los efectos sobre la actitud hacia las drogas de diferentes estrategias de prevención específica y de promoción de la salud en general. Se espera que los programas activos produzcan una actitud más favorable a la abstinencia de las drogas frente a un grupo de atención placebo y un grupo que no recibe intervención, y que los programas específicos obtengan un efecto mayor que el programa inespecífico.

\section{Método}

\section{Participantes}

Los participantes fueron 133 alumnos de tercer curso de ESO, 63 chicos (47.4\%) y 70 chicas $(52.6 \%)$, con un rango de edad de 13 a 15 años $(\mathrm{M}=14.17$; DT $=.72)$. El nivel socioe- 
conómico era medio en el $82 \%$ de los casos y la mayor parte $(95 \%)$ era de nacionalidad española. Por medio de la prueba $\chi^{2}$ de homogeneidad de la distribución de frecuencias, se comprobó que no existían diferencias estadísticamente significativas de género y edad entre los grupos $\left(\chi^{2}=0.10 ; p=.77\right)$.

\section{Instrumentos}

Cuestionario de Consumo de Drogas (Espada, Méndez e Hidalgo, 2003). Se trata de un cuestionario que evalúa la frecuencia y la intensidad del consumo de diferentes sustancias. Está formado por 13 ítems de alternativa múltiple.

Cuestionario de Intención de Consumo (Espada, Méndez e Hidalgo, 2003). Está compuesto por siete ítems con formato de respuesta dicotómico, solicitando el juicio de los sujetos sobre su intención de realizar conductas relacionadas con la compra, consumo ocasional o habitual de drogas. Se realizó un análisis de la dimensionalidad del test mediante un análisis factorial, hallando una estructura factorial unidimensional. El coeficiente de consistencia interna, alfa de Cronbach, fue de .66, satisfactorio teniendo en cuenta el reducido número de ítems que componen la escala.

Cuestionario de Actitudes hacia las Drogas (Escámez, 1990). La versión inicial de este cuestionario consta de 23 ítems con una escala de respuesta graduada de 1 a 5 . Tras su aplicación en el pretest se depuró la prueba, siendo eliminados 5 ítems por su baja correlación ítem-test. Se estudió la estructura factorial del cuestionario, que presentó un coeficiente de fiabilidad de .72 .

\section{Procedimiento}

La evaluación y la intervención se realizaron en el contexto escolar. Previamente al inicio de la intervención se informó mediante una circular a los padres, solicitando su consentimiento. Se realizó una evaluación pretest en dos sesiones, en la que se informó a los participantes de los objetivos del estudio y se garantizó la confidencialidad y el anonimato de las respuestas. Una semana más tarde se inició la intervención. Las sesiones se desarrollaban dos veces por semana durante el horario escolar. En la semana posterior a la finalización del pro- 
grama se realizó la evaluación postest. A los tres meses tuvo lugar el primer seguimiento, y al cabo de seis meses se realizó un segundo seguimiento.

Al tratarse de un estudio piloto cuasi-experimental, cada grupo de clase fue asignado aleatoriamente a cada una de las cinco condiciones. El grupo Programa Saluda (PS) estuvo compuesto por 35 sujetos, el grupo Life Skills Training (LST) por 39 sujetos, el grupo Programa de Educación para la Salud (EPS) lo formaron 21 sujetos, y el grupo Atención Placebo (AP), por 16 sujetos. En el grupo de atención placebo se realizaron diez sesiones de debate en grupo, realizando el monitor funciones de moderador en las discusiones. El grupo control sin intervención (GC) con 22 sujetos funcionó como control puro (control inactivo).

El Programa Saluda (Espada y Méndez, 2003) se centra en el consumo de drogas en el contexto del ocio. Parte de un modelo teórico que explica el uso y abuso de drogas en función de variables de carácter personal y social. A partir del análisis motivacional del consumo, el programa incluye los siguientes componentes principales: entrenamiento en resolución de problemas, fomento de las actividades de ocio saludable, análisis de la publicidad, entrenamiento en habilidades sociales, información sobre las drogas y sus efectos, compromiso público y entrenamiento en autorrefuerzo. El programa consta de diez sesiones de una hora.

El programa Life Skills Training (Botvin, 1998) ha sido calificado como uno de los mejores entre más de 300 programas de prevención (Price, Cowen Lorion y Ramos-McKay, 1988). El programa ha sido aplicando con evaluaciones muy positivas, según muestran los estudios de evaluación (Botvin y Eng, 1982; Botvin, Baker, Filazzola y Botvin, 1990; Botvin, Schinke, Epstein y Díaz, 1994; Botvin, Griffin, Diaz, Scheier, Williams y Epstein, 2000; Botvin, Griffin, Diaz y Ifill-Williams, 2001) y los estudios meta-analíticos (Lence, Gómez y Luengo, 1997). Los resultados en cuanto a reducción de consumo han llegado a ser del 80\% en tabaco y alcohol. En España el programa fue traducido y adaptado por Luengo, Gómez, Garra, Romero y Otero-López (1998), que incorporaron nuevos contenidos. En esta aplicación no fueron incluidos dichos contenidos adicionales.

El programa de Educación para la Salud es una intervención basada en técnicas conductuales (transmisión de información, entrenamiento en habilidades, entrenamiento en resolución de problemas y autorreforzamiento), similares a las empleadas en los dos programas anteriores. Para su elaboración se adaptaron los contenidos del programa Saluda, eliminando 
las referencias explícitas a las drogas. Las sesiones informativas versaron sobre la salud y los hábitos de vida saludables. Se entrenaron habilidades aplicadas a todo tipo de situaciones excepto el consumo de drogas. La intervención constó de diez sesiones de una hora.

\section{Análisis de datos}

En primer lugar se realizaron análisis descriptivos para obtener las frecuencias y puntuaciones medias en las distintas escalas. Para la comparación de medias se calcularon ANOVAS, y para el análisis de las diferencias significativas se realizaron pruebas post hoc para la comparación de grupos dos a dos (HSD de Tukey). En un análisis posterior se obtuvieron los índices del tamaño del efecto. Para determinar si las intervenciones preventivas mejoran respecto a la no intervención, se calculó la magnitud de la diferencia entre cada grupo de tratamiento con el grupo sin tratamiento. En segundo lugar, a fin de conocer si se daban efectos inespecíficos a causa de las intervenciones preventivas, se calculó la magnitud de la diferencia entre cada grupo de tratamiento con el grupo de atención placebo. Para conocer los efectos diferenciales entre las intervenciones, se calculó la magnitud de la diferencia entre los tres programas activos. Cada índice "d" se obtuvo restando las medias de los dos grupos implicados y dividiendo por una estimación de la desviación típica conjunta a partir de las de los dos grupos. Según la clasificación de Cohen (1988), un valor negativo del índice d supone un efecto de empeoramiento con el tratamiento, un valor de .20 significa un efecto bajo, un valor de .50 un efecto medio y un .80 un efecto elevado. Por último, se calculó la potencia estadística empleando el programa G*Power 3.1 (Faul, Erdfelder, Buchner y Lang (2009).

\section{Resultados}

\section{Intención de consumo}

Mientras que en el pretest la intención de beber era similar en todos los grupos, en el postest aparecen diferencias significativas $\left[\mathrm{F}_{(4.92)}=2.85, p<.05\right]$. Los grupos EPS y GC aumentan en intención de consumo, mientras que el grupo PS se mantiene y los grupos LST y AP disminuyen ligeramente. Las diferencias son significativas entre el programa LST $(\mathrm{M}=$ 1.85) y el programa EPS $(\mathrm{M}=2.37)(p<.05)$. En el primer seguimiento aparecen diferencias marginalmente significativas $\left[\mathrm{F}_{(4.92)}=2.28, p=.06\right]$ entre $\mathrm{PS}(\mathrm{M}=1.76)$ y $\mathrm{GC}(\mathrm{M}=2.50, p<$ 
$.05)$. En el segundo seguimiento existen diferencias significativas $\left[\mathrm{F}{ }_{(4.89)}=2.76, p<.05\right]$, observándose en las comparaciones dos a dos diferencias entre PS ( $\mathrm{M}=1.81)$ y GC $(\mathrm{M}=$ 2.57, $p<.05)$. La tendencia en la intención de consumo a largo plazo es a aumentar en el GC y a mantenerse o disminuir en los restantes grupos (Tabla 1).

Tabla 1. Intención de beber en un futuro

\begin{tabular}{lrrrrrrc}
\hline & PS & LST & EPS & AP & GC & F & Sig. \\
\hline Pre-test & & & & & & & \\
Media & 2.00 & 2.00 & 2.00 & 2.12 & 2.00 & 2.06 & n.s \\
Desviación típica & .81 & .76 & .82 & .96 & .82 & & \\
Post-test & & & & & & & \\
Media & 2.00 & 1.85 & 2.37 & 2.07 & 2.37 & 2.86 & .03 \\
Desviación típica & .97 & .57 & .68 & .70 & .68 & & \\
Seg 1 & & & & & & & \\
Media & 1.76 & 2.00 & 2.50 & 2.19 & 2.50 & 2.28 & .07 \\
Desviación típica & .70 & .66 & .65 & .91 & .65 & & \\
Seg 2 & & & & & & & \\
Media & 1.81 & 1.95 & 2.57 & 2.06 & 2.57 & 2.77 & .03 \\
Desviación típica & .75 & .72 & .65 & .57 & .65 & & \\
\hline
\end{tabular}

PS: Programa Saluda; LST: Programa Life Skills Training; EPS: Programa de Promoción de la Salud; AP: Atención-Placebo; GC: No Intervención; Seg 1: seguimiento a los 3 meses; Seg 2: seguimiento a los 6 meses.

En cuanto a la intención de fumar (Tabla 2), en el pretest todos los grupos parten de la misma puntuación a excepción del grupo PS con una puntuación algo más elevada, a pesar de ello sin la presencia de diferencias significativas. Aparecen cambios a corto plazo, pero con el tiempo no se presentan cambios significativos. En el postest se observan diferencias globales marginalmente significativas $\left[\mathrm{F}_{(4.92)}=2.27, p=.06\right]$. En el seguimiento a los tres meses no aparecen diferencias significativas, mientras que a los seis meses hay tendencia a la significación entre PS y GC $(p=.09)$. En el seguimiento, tanto a los tres como a los seis meses, no aparecen diferencias significativas entre los grupos.

Respecto a la intención de consumir cannabis (Tabla 3), en el postest se observan diferencias significativas entre los grupos $\left[\mathrm{F}_{(4.91)}=2.77, p=.03\right]$. En el seguimiento a los tres meses la intención aumenta en todos los grupos, con diferencias globales significativas $\left[\mathrm{F}_{(4.91)}\right.$ $=2.93, p<.05]$. Hay diferencias entre PS $(\mathrm{M}=2.42)$ frente a AP $(\mathrm{M}=2.00, p<.05)$ y a $\mathrm{GC}$ $(\mathrm{M}=1.36, p<.05)$. En el seguimiento a los seis meses se producen diferencias significativas $\left[\mathrm{F}_{(4.89)}=3.16, p<.05\right]$. 
Tabla 2. Intención de fumar tabaco en un futuro

\begin{tabular}{lccccccc}
\hline & PS & LST & EPS & AP & GC & F & Sig. \\
\hline Pre-test & & & & & & & \\
Media & 2.21 & 2.00 & 2.00 & 2.00 & 2.00 & 1.47 & n.s \\
Desviación típica & 1.18 & .97 & 1.14 & 1.41 & .82 & & \\
Post-test & & & & & & & \\
Media & 2.42 & 2.05 & 2.05 & 2.00 & 1.36 & 2.27 & .06 \\
Desviación típica & 1.30 & 1.12 & 1.27 & 1.36 & .73 & & \\
Seg 1 & & & & & & & \\
Media & 2.46 & 2.04 & 2.04 & 1.81 & 1.43 & 1.84 & n.s \\
Desviación típica & 1.33 & 1.12 & 1.29 & 1.22 & .81 & & \\
Seg 2 & & & & & & & \\
Media & 2.21 & 2.04 & 1.90 & 1.94 & 1.97 & 1.03 & n.s \\
Desviación típica & 1.25 & 1.09 & 1.18 & 1.43 & .93 & & \\
\hline
\end{tabular}

PS: Programa Saluda; LST: Programa Life Skills Training; EPS: Programa de Promoción de la Salud; AP: Atención-Placebo; GC: No Intervención; Seg 1: seguimiento a los 3 meses; Seg 2: seguimiento a los 6 meses.

Tabla 3. Intención de consumir cannabis en un futuro

\begin{tabular}{lccccccc}
\hline & PS & LST & EPS & AP & GC & F & Sig. \\
\hline Pre-test & & & & & & & \\
Media & 1.26 & 1.14 & 1.48 & 1.00 & 1.14 & 1.77 & n.s \\
Desviación típica & .65 & .64 & .75 & .00 & .35 & & \\
Post-test & & & & & & & \\
Media & 1.42 & 1.14 & 1.45 & 1.00 & 1.04 & 2.77 & .03 \\
Desviación típica & .69 & .65 & .68 & .00 & .22 & & \\
Seg 1 & & & & & & & \\
Media & 2.42 & 2.04 & 2.05 & 2.00 & 1.36 & 2.93 & .02 \\
Desviación típica & 1.30 & 1.12 & 1.28 & 1.36 & .53 & & \\
Seg 2 & & & & & & & \\
Media & 1.57 & 1.09 & 1.24 & 1.06 & 1.05 & 3.16 & .02 \\
Desviación típica & .94 & .43 & .44 & .25 & .22 & & \\
\hline
\end{tabular}

PS: Programa Saluda; LST: Programa Life Skills Training; EPS: Programa de Promoción de la Salud; AP: Atención-Placebo; GC: No Intervención; Seg 1: seguimiento a los 3 meses; Seg 2: seguimiento a los 6 meses.

Si observamos la magnitud del tamaño del efecto (Tabla 4) para la variable intención de beber alcohol, a corto plazo el grupo PS se muestra más eficaz que los grupos GC y EPS con un tamaño del efecto bajo y moderado respectivamente. A su vez, a corto plazo el programa LST también se muestra superior a los programas GC y EPS con efectos altos y que el programa AP con una magnitud baja. Sin embargo, a largo plazo el programa PS presenta un tamaño del efecto alto al compararlo con el grupo control (GC), moderado con respecto al grupo EPS, bajo con el grupo LST, y moderado (primer seguimiento) y bajo (segundo seguimiento) al compararlo con el grupo AP. 
Sobre la intención de fumar, los tamaños del efecto son nulos o negativos en el postest y el seguimiento para todos los grupos. Del mismo modo, estos mismos resultados se muestran para la variable intención de consumo de cannabis, con excepción del grupo LST mostrándose superior al grupo EPS con una magnitud del tamaño baja.

Tabla 4. Magnitud del tamaño del efecto y potencia estadística en la variable intención de consumo.

\begin{tabular}{|c|c|c|c|c|c|c|c|c|c|c|c|c|c|c|c|c|c|c|}
\hline & \multicolumn{2}{|c|}{$\begin{array}{l}\text { PS- } \\
\text { GC }\end{array}$} & \multicolumn{2}{|c|}{$\begin{array}{c}\text { LST- } \\
\text { GC }\end{array}$} & \multicolumn{2}{|c|}{$\begin{array}{c}\text { EPS- } \\
\text { GC }\end{array}$} & \multicolumn{2}{|c|}{$\begin{array}{l}\text { PS- } \\
\text { AP }\end{array}$} & \multicolumn{2}{|c|}{$\begin{array}{c}\text { LST- } \\
\text { AP }\end{array}$} & \multicolumn{2}{|c|}{$\begin{array}{c}\text { EPS- } \\
\text { AP }\end{array}$} & \multicolumn{2}{|c|}{$\begin{array}{l}\text { PS- } \\
\text { LST }\end{array}$} & \multicolumn{2}{|c|}{$\begin{array}{l}\text { PS- } \\
\text { EPS }\end{array}$} & \multicolumn{2}{|c|}{ LST-EPS } \\
\hline & $\mathrm{d}$ & $P$ & $\mathrm{~d}$ & $P$ & $\mathrm{~d}$ & $P$ & $\mathrm{~d}$ & $P$ & $\mathrm{~d}$ & $P$ & $\mathrm{~d}$ & $P$ & $\mathrm{~d}$ & $P$ & $\mathrm{~d}$ & $\mathrm{P}$ & $\mathrm{d}$ & $P$ \\
\hline $\begin{array}{l}\text { Post-test } \\
\text { Alcohol }\end{array}$ & .45 & .99 & .83 & 1 & -.28 & .91 & .08 & .15 & .35 & .98 & -.63 & 1 & -.19 & .60 & .63 & 1 & .97 & 1 \\
\hline Tabaco & -.1 .03 & 1 & -.73 & 1 & -.67 & 1 & -.32 & .97 & -.04 & .07 & -.04 & .07 & -.31 & .96 & -.29 & .93 & -.00 & .05 \\
\hline Cannabis & -.77 & 1 & -.21 & .69 & -.82 & 1 & -.82 & 1 & -.28 & .91 & -.88 & 1 & -.42 & .99 & .04 & .07 & .47 & .47 \\
\hline $\begin{array}{c}\text { Seg 1 } \\
\text { Alcohol }\end{array}$ & 1.10 & 1 & .76 & 1 & .55 & 1 & .65 & 1 & .30 & .94 & .08 & .15 & .35 & .98 & .56 & 1 & .21 & .69 \\
\hline Tabaco & -.97 & 1 & -.62 & 1 & -.57 & 1 & -.51 & .99 & -.20 & .64 & -.18 & .55 & -.35 & .98 & -.32 & .97 & -.00 & .05 \\
\hline Cannabis & -1.05 & 1 & -.72 & 1 & -.66 & 1 & -.32 & .97 & -.03 & .06 & -.04 & .07 & -.32 & .97 & -.29 & .93 & .01 & .10 \\
\hline $\begin{array}{l}\text { Seg } 2 \\
\text { Alcohol }\end{array}$ & 1.09 & 1 & .90 & 1 & .46 & .99 & .38 & .99 & .17 & .50 & -.23 & .77 & .19 & .60 & .53 & .99 & .36 & .99 \\
\hline Tabaco & -.22 & .82 & -.07 & .12 & .07 & .12 & -.20 & .64 & -.08 & .15 & .03 & .06 & -.15 & .41 & -.26 & .86 & -.12 & .28 \\
\hline Cannabis & -.82 & 1 & -.12 & .28 & -.55 & 1 & -.74 & 1 & -.08 & .15 & -.49 & .99 & -.70 & 1 & -.47 & .99 & .34 & .98 \\
\hline
\end{tabular}

PS: Programa Saluda; LST: Programa Life Skills Training; EPS: Programa de Promoción de la Salud; AP: AtenciónPlacebo; GC: No Intervención; Seg 1: seguimiento a los 3 meses; Seg 2: seguimiento a los 6 meses.

\section{Actitud hacia las drogas}

En la tabla 5 se presentan las medias en la escala de actitudes en los cuatro momentos de evaluación. Mientras que en el pretest la actitud hacia el consumo de drogas era similar en todos los grupos, a excepción del grupo AP con puntuaciones mayores, en el postest varían significativamente $\left[\mathrm{F}_{(4.90)}=3.105 ; p<.05\right]$. Estas variaciones se presentan principalmente entre el grupo PS y EPS. Sin embargo, en ambos seguimientos no se producen diferencias significativas.

En cuanto a las comparaciones post hoc dos a dos para la variable actitud ante las drogas, la tabla 6 muestra magnitudes altas en el postest del programa PS frente a los programas GC y EPS, y moderadas respecto a AP y LST. Del mismo modo, el programa LST se muestra superior al EPS con un tamaño del efecto moderado en postest. Si nos centramos en los resultados hallados en el seguimiento, es el grupo PS el único que se muestra superior al resto en lo que respecta a eficacia. A los tres meses el grupo PS con un efecto bajo se muestra superior 
al grupo control y, tanto a los tres meses como a los seis, frente a LST y EPS con un efecto moderado.

Tabla 5. Medias y desviaciones típicas en actitud hacia las drogas.

\begin{tabular}{lrrrrrrc}
\hline & PS & LST & EPS & AP & GC & F & Sig. \\
\hline Pre-test & & & & & & & \\
Media & 71.14 & 70.41 & 71.19 & 74.12 & 71.53 & 1.04 & n.s \\
Desviación típica & 8.80 & 10.22 & 9.43 & 9.49 & 10.57 & & \\
Post-test & & & & & & & \\
Media & 77.48 & 69.95 & 62.05 & 72.28 & 69.28 & 3.10 & .02 \\
Desviación típica & 9.21 & 12.64 & 18.32 & 10.56 & 9.23 & & \\
Seg 1 & & & & & & & \\
Media & 75.00 & 69.14 & 69.90 & 74.27 & 70.18 & 1.02 & n.s \\
Desviación típica & 10.71 & 11.25 & 12.18 & 12.20 & 13.64 & & \\
Seg 2 & & & & & & & \\
Media & 75.27 & 69.81 & 68.90 & 75.31 & 74.19 & 1.55 & n.s \\
Desviación típica & 10.60 & 11.58 & 14.19 & 9.01 & 7.40 & & \\
\hline
\end{tabular}

PS: Programa Saluda; LST: Programa Life Skills Training; EPS: Programa de Promoción de la Salud; AP: Atención-Placebo; GC: No Intervención; Seg 1: seguimiento a los 3 meses; Seg 2: seguimiento a los 6 meses.

Tabla 6. Magnitud del tamaño del efecto y potencia estadística en actitud hacia las drogas.

\begin{tabular}{|c|c|c|c|c|c|c|c|c|c|c|c|c|c|c|c|c|c|c|}
\hline & \multicolumn{2}{|c|}{$\begin{array}{l}\text { PS- } \\
\text { GC }\end{array}$} & \multicolumn{2}{|c|}{$\begin{array}{l}\text { LST- } \\
\text { GC } \\
\end{array}$} & \multicolumn{2}{|c|}{ EPS-GC } & \multicolumn{2}{|c|}{$\begin{array}{l}\text { PS- } \\
\text { AP }\end{array}$} & \multicolumn{2}{|c|}{$\begin{array}{c}\text { LST- } \\
\text { AP } \\
\end{array}$} & \multicolumn{2}{|c|}{$\begin{array}{c}\text { EPS- } \\
\text { AP }\end{array}$} & \multicolumn{2}{|c|}{$\begin{array}{l}\text { PS- } \\
\text { LST }\end{array}$} & \multicolumn{2}{|c|}{$\begin{array}{l}\text { PS- } \\
\text { EPS }\end{array}$} & \multicolumn{2}{|c|}{$\begin{array}{l}\text { LST- } \\
\text { EPS }\end{array}$} \\
\hline & $\mathrm{d}$ & $P$ & $\mathrm{~d}$ & $\mathrm{P}$ & $\mathrm{d}$ & $P$ & $\mathrm{~d}$ & $\mathrm{P}$ & $\mathrm{d}$ & $\mathrm{P}$ & d & $\mathrm{P}$ & $\mathrm{d}$ & $\mathrm{P}$ & d & $P$ & d & $\mathrm{P}$ \\
\hline Post-test & .89 & 1 & .06 & .17 & -.50 & .99 & -.53 & 1 & -.20 & .75 & -.66 & 1 & .67 & 1 & 1.05 & 1 & .50 & .99 \\
\hline Seg 1 & .39 & .99 & -.08 & .23 & -.02 & .07 & .06 & .17 & -.44 & .99 & -.36 & .99 & .53 & 1 & .44 & .99 & -.06 & .17 \\
\hline Seg 2 & .12 & .39 & -.45 & .99 & -.47 & .99 & .00 & .05 & -.52 & 1 & -.52 & 1 & .49 & .99 & .50 & .99 & .07 & .20 \\
\hline
\end{tabular}

PS: Programa Saluda; LST: Programa Life Skills Training; EPS: Programa de Promoción de la Salud; AP: AtenciónPlacebo; GC: No Intervención; Seg 1: seguimiento a los 3 meses; Seg 2: seguimiento a los 6 meses.

\section{Discusión y Conclusiones}

El objetivo de este trabajo fue valorar los efectos de tres intervenciones preventivas sobre la actitud hacia las drogas en una muestra de adolescentes de Secundaria. Se ha demostrado que las intervenciones específicas, en comparación con la no específica (educación para la salud) son más eficaces para conseguir promover actitudes favorables al no consumo de sustancias e influir sobre la intención de consumo de alcohol. En la intención de consumo de bebidas alcohólicas, a corto plazo el programa Life Skills Training disminuyó la intención de 
consumo y el grupo Saluda no provocó cambios. Sin embargo, a largo plazo si redujo significativamente la intención de consumir. Al comparar ambos programas, los resultados revelaron un efecto mayor del programa Saluda sobre la intención de consumir alcohol a largo plazo (primer seguimiento) con una baja magnitud del tamaño y sobre las actitudes con un efecto moderado tras la intervención y el seguimiento de ésta.

Un objetivo adicional consistió en comparar ambas intervenciones activas (Programa Saluda y Life Skills Trainig) con un programa preventivo de promoción de la salud en general (Programa de Educación para la Salud), observando si la educación para la salud en general era tan eficaz como un programa específico para modificar la actitud hacia las drogas y su consumo. Los dos programas activos produjeron mayores cambios en las actitudes y en la intención de consumir alcohol que el programa de Educación para la Salud. Sobre la intención de consumo de alcohol, con un tamaño del efecto moderado, tanto a corto como a largo plazo el programa Saluda se muestra superior a la intervención de educación para la salud. Para esta misma variable, el programa Life Skills Training a corto y largo plazo también se mostró más eficaz que el grupo Educación para la Salud. Sobre la actitud hacia el consumo, el programa Saluda presenta efectos más potentes tanto a corto como a largo plazo frente al programa Educación para la Salud. Por su parte, el programa Life Skills Training se muestra superior al grupo Educación para la Salud en el postest con un tamaño del efecto moderado.

Estos resultados revelan el mayor potencial, en cuanto a eficacia preventiva se refiere, de los programas diseñados específicamente para prevenir el consumo de drogas. Una revisión de 214 intervenciones de promoción de la salud en jóvenes españoles desde el año 1995 hasta el 2000, reveló que los programas dirigidos a la reducción del tabaquismo y/o alcohol, mostraron eficacia para disminuir el consumo de tabaco y en dos de las cuatro intervenciones se consiguió retrasar la edad de inicio al consumo de tabaco. En los programas en los que se abordaron otras drogas ilegales como el cannabis, solo se había evaluado el proceso (Hernán, Ramos y Fernández, 2001). A diferencia de estos resultados, una revisión sistemática realizada en Reino Unido concluye que no existe todavía suficiente evidencia sobre la eficacia de los programas dirigidos a prevenir el consumo de drogas ilegales (White y Pitts, 1998). Los resultados de este estudio confirman la necesidad de mejorar las evaluaciones de las intervenciones en promoción de la salud. En cualquier caso no debemos obviar la influencia de estos programas sobre la mejora del bienestar y la calidad de vida de los adolescentes. 
Otro aspecto a destacar sobre las evaluaciones realizadas en este estudio son las escasas diferencias significativas encontradas entre los grupos experimentales y control en el pretest. Los grupos pueden considerarse equivalentes, tanto en las variables de consumo como en la variable actitud hacia las drogas, salvando así el problema de la falta de equivalencia de los grupos que citan Flay y Best (1982).

Al comparar los tres programas preventivos con el grupo que no recibió intervención, los programas Saluda y Life Skills Training se mostraron más eficaces que el Grupo Control para disminuir la intención de beber. Tan solo el programa Saluda mejoró las actitudes frente al consumo. Por el contrario, el programa Educación para la Salud no produjo diferencias significativas respecto al Grupo Control. Por tanto, la evidencia indica que la intervención es mejor que la no intervención y que es preciso continuar trabajando en la mejora de las intervenciones preventivas.

Con respecto a las diferencias intergrupo e intragrupo para las variables intención de consumo de tabaco y cannabis, fueron nulas y negativas. En consumo de cannabis se encuentra una pequeña diferencia en la que el programa Life Skills Training se muestra un mejor, en términos de eficacia preventiva, que el programa de Educación para la Salud con un tamaño del efecto bajo $(\mathrm{d}=.34)$. Estos datos muestran como estos programas, en concreto Saluda y Life Skills Training, resultan más eficaces para influir sobre la variable intención de consumo de alcohol que sobre la intención de consumo de tabaco o cannabis. Este resultado nos lleva a dirigir la atención sobre la especificidad de los programas en función de las sustancias sobre las que incide el programa preventivo. En cualquier caso, las intervenciones más eficaces como la de Casas (1998), Alonso y Del Barrio (1998) o Espada (2002) incluían entre sus objetivos la prevención del consumo de alcohol.

Con respecto a las comparaciones de los tres programas principales con el grupo que recibe Atención-Placebo, el programa Saluda (largo plazo) y Life Skills Training (corto plazo y primer seguimiento) se mostraron más eficaces frente al grupo Atención-Placebo que el programa de Educación para la Salud.

Tras los resultados de este estudio se concluye que el programa Saluda produce la mayor disminución en intención de consumo de alcohol a largo plazo. No obstante, ninguna de las intervenciones se mostró capaz de disminuir la intención de consumo de tabaco y canna- 
bis. Por otro lado, el programa Life Skills Training fue el segundo mejor programa para influir sobre la intención de no consumo de bebidas. No se hallaron diferencias entre esta intervención y el resto para mejorar las actitudes frente al consumo, a excepción de una mejora frente al grupo de Educación para la Salud a corto plazo. Sin embargo, el programa de Educación para la Salud no se mostró eficaz ni para disminuir la intención de consumo ni para mejorar las actitudes frente a las drogas tanto por sí solo como si es comparado con el resto de intervenciones.

Teniendo en cuenta la literatura previa, podemos confirmar como aquellas actitudes desfavorables al consumo de sustancias se relacionan con expectativas negativas por parte de los individuos hacia el consumo tal como demuestran estudios como el de Grube y Agostinelli (1999).

Entre las limitaciones de esta investigación cabe destacar que, al tratarse de un estudio piloto, el tamaño muestral es reducido, lo que puede afectar a la generalización de los resultados. No obstante, si comparamos el tamaño muestral de este estudio con el de otros estudios nacionales, observamos que no se diferencia demasiado del promedio. La evaluación a más largo plazo también puede ser un aspecto a mejorar, debido a que en el área de la prevención de la drogodependencia los resultados de las intervenciones pueden observarse mejor a largo plazo (Bangert-Drowns, 1988). En este estudio se estableció un periodo de seis meses como último seguimiento, debido a que la finalización del curso escolar y los cambios de centro dificultaba el seguimiento de los sujetos.

Respecto a las sustancias sobre las que se centraron las intervenciones, los programas se mostraron más eficaces para influir sobre la intención de consumo de alcohol que sobre tabaco y cannabis. De cualquier modo, es razonable que los programas en cierto modo se muestren más potentes sobre esta sustancia teniendo en cuenta que el alcohol es por excelencia una de las sustancias más consumidas entre los adolescentes de estas edades (PND, 2008) y se tenga en cuenta principalmente sobre los contenidos de los programas preventivos. En este sentido, los programas presentados en este estudio se centran más sobre esta sustancia que sobre otras. Si bien es cierto, que no debemos olvidar que los porcentajes de consumo de tabaco también son alarmantes, existen programas específicos para este fin ninguno incluido en este estudio. 
Estos resultados animan a continuar investigando sobre la eficacia de los programas de prevención escolar ya que, a pesar de las limitaciones se producen cambios importantes en las actitudes ante las drogas que se consideran de peso como mediadoras del consumo. Aunque es preferible planificar la prevención del abuso de drogas como una intervención continuada en el contexto de la educación para la salud, los programas escolares intensivos presentan la ventaja de que suponen una forma efectiva de lograr cambios cognitivos y de enseñar a los alumnos habilidades conductuales que pueden aplicar en situaciones de oferta de drogas y también en otras áreas de la vida. A pesar de estas limitaciones, este estudio aporta indicios interesantes sobre la eficacia diferencial de distintas estrategias preventivas para favorecer las actitudes pro-salud de los adolescentes y reducir la intención de consumo de alcohol.

\section{Referencias}

Alonso, C. y Del Barrio, V. (1997). Efectividad de tres intervenciones para la prevención del consumo de tabaco en el medio escolar. Revista de Psicología General y aplicada, 50 (2), 223-241.

Alonso, C. y Del Barrio, V. (1998). Efectividad de tres intervenciones para la prevención del consumo de alcohol en la escuela. Análisis y Modificación de Conducta, 24 (97), 679701.

Bangert-Drowns, R. L. (1988). Effects of school-based substance abuse education: Ametaanalysis. Journal of Drug Education, 18, 243-264.

Casas, J. (1998). Actitudes, habilidades sociales y autocontrol en la prevención del consumo abusivo de alcohol y tabaco. Tesis Doctoral sin publicar. Universidad Autónoma de Madrid.

Cava, M. J., Musitu, G., \& Murgui, S. (2006). Familia y violencia escolar: El rol mediador de la autoestima y la actitud hacia la autoridad institucional. Psicothema, 18, 367-373.

Cohen, J. (1988). Statistical power analysis for the behavioral sciences (2a ed.). Hillsdale, NH: Erlbaum.

Colder, C., Campbell, R., Ruel, E., Richardson, J.L. y Flay, B. (2002). A finite mixture model of growth trajectories of adolescent alcohol use: Predictors and consequences. Journal of Consulting and Clinical Psychology, 70 (4), 976-985. 
DeBills, M. D., Clark, D. B., Beers, S. R., Soloff, P. H., Boring, A. M., Hall, J., Kersh, A. y Keshavan, M. M. (2000). Hippocampal volume in adolescent-onset alcohol use disorder. American Journal of Psychiatry, 157, 737-744.

Espada, J. P. (2002). Eficacia de tres intervenciones para prevenir el abuso de alcohol y drogas de síntesis en el contexto escolar. Tesis doctoral. Universidad de Murcia.

Espada, J. P., Méndez, F. X. e Hidalgo, M. D. (2000). Consumo de alcohol en escolares: descenso de la edad de inicio y cambios en los patrones de ingesta. Adicciones, 12 (1), $57-64$

Espada, J. P., Méndez, F. X., Griffin, K. W. y Botvin, G. J. (2003). Adolescencia: consumo de alcohol y otras drogas. Papeles del Psicólogo, 84, 9-17.

Espada, J. P., Méndez, X., Botvin, G. J., Griffin, K. W., Orgilés, M. y Rosa, A.I. (2002). ¿Éxito o fracaso de la prevención del abuso de drogas en el contexto escolar? Un meta-análisis de los programas en España. Psicología Conductual, 10 (3), 581-602.

European Monitoring Centre for Drugs and Drug Addiction (2006). The state of the drugs problem in Europe. Lisboa: European Monitoring Centre for Drugs and Drug Addiction.

Faul, F., Erdfelder, E., Buchner, A., y Lang, A. G. (2009). Statistical power analyses using G*Power 3.1: Tests for correlation and regression analyses. Behavior Research Methods, 41, 1149-1160.

Fishbein, M. y Azjen, I. (1975). Belief, attitude and behaviour: An introduction to theory and research. Reading, MA: Addison-Wesley.

Flay, B. R. y Best, J. A. (1982). Overcoming design problems in evaluating health behavior programs. Evaluation and the Health Professions, 5 (1), 43-69.

Golberg, J. H., Halpern-Felsher, B. L. y Millstein, S. G. (2002). Beyond invulnerability: The importance of benefits in adolescents decision to drink alcohol. Health Psychology, $21(5), 477-484$.

Grube, J. W. y Angostinelli, G. E. (1999). Perceived consequences and adolescent drinking: Nonlinear and interactive models of alcohol expectancies. Psychology of Addictive Behaviors, 13(4), 303-312.

Hawkins, J. D., Catalano, R. F. y Miller, J. Y. (1992). Risk and protective factors for alcohol and other drug problems in adolescence and early adulthood: implications for substance abuse prevention. Psychological Bulletin, 112, 64-105. 
Hernán, M., Ramos, M. y Fernández, A. (2001). Revisión de los trabajos publicados sobre promoción de la Salud en jóvenes españoles. Revista Española de Salud Pública, 75, 491-504.

Masten, A. y Faden, V. (2008). Underage Drinking: A Developmental Framework. Pediatrics, 121, 235-251.

Observatorio Español sobre Drogas (2008). Encuesta sobre drogas a población escolar. Madrid: Delegación del Gobierno para el Plan Nacional Sobre Drogas, Ministerio del Interior.

Salvador, T. y Martínez, I. M. (1997). La evaluación de programas de prevención en las drogodependencias. Revista de Estudios de Juventud, 40, 95-108.

Turrisi, R. y Wiersma, K. A. (1999). Examination of judgments of drunkenness, binge drinking, and drunk driving tendencies in teens with and without a family history of alcohol abuse. Alcoholism: Clinical and Experimental Research, 23, 1-8.

White D, y Pitts M. (1998). Educating young people about drugs: a systematic review. Addiction, 93(10), 1475-1487. 